\title{
The Application of Minimalism in Modern Packaging Design
}

\author{
Liu Jinglong \\ Shandong University of Art \& Design, Changqing, Jinan, China
}

1263556797@qq.com

Keywords: Minimalism, Packaging Design, Application, Principles

\begin{abstract}
Minimalism is a popular art style in the design field at present. It has been applied to various fields, including packaging design. By analyzing the characteristics and development of minimalism and the problems and trends in modern packaging design.This paper tries to explore the minimalist principles of modern packaging design application.
\end{abstract}

Minimalism is an art trend and style formed around World War II. It was first formed in the field of architecture and interior design and then it gradually affected the furniture design, graphic design, industrial product design and other fields. What's more, Minimalism has gradually become a fashion trend which is not only gradually being a culture but also gradually being a kind of aesthetic definition and philosophical education. As an exploration of idealism, minimalism has formed various theories. More researchers combine it with other art forms and business forms. More and more people begin to focus on packaging design after the extensive exploration in the field of architecture, plane and product.

\section{The Concept and Main Characteristics of Minimalism}

In the early twentieth century, the West produced a modernist school which had great influence on the later generations. In terms of architectural design, the modernist school emphasizes the features of "function first, form second"," decoration is evil"and "cost budget". Later, these features were also adopted in other fields including interior design, furniture design, and product design, resulting in a style of "formless" - Minimalism. The main idea of minimalism comes from the famous motto of modernist architect Ludwig Mies Van der Rohe:"less is more." In addition to emphasizing "function", minimalism also minimizes the design elements, colors, lighting and raw materials. Because of this, minimalist style works are very introverted and minimalists can achieve the effect of "less is more". Besides, minimalists also adopt advanced technology constantly and pursue original materials and forms in their works. The so-called simplification of minimalism is not a simple cut but the integration of materials and colors and structures after designers' careful consideration. Minimalism tries to achieve the maximize performance with minimal material and design elements. The style of minimalism not only extends the style of modernism but also adopts contemporary science and technology and Eastern and Western cultures. It has a strong inclusiveness.

\section{The Background and History of Minimalism}

In nineteenth century, the design has become mature. It is due to not only the Industrial Revolution and the development of machinery but also the foreign exchange of western countries and the liberation of human rights.Meanwhile, it is also benefited from the development of science and the rise of the genre of humanism. After the Crystal Palace Expo, in order to explore the relationship between technology and art, the designers promote design movements or genres,like The Arts \& Crafts Movement, Art Nouveau, Art Deco, Modernism Design and Post Modernism. The modern school of design represented by Bauhaus is the mature period of industrial design, and he emergence of masters such as Walter Gropius, Ludwig Mies Van der Rohe and Le Corbusier has 
caused a great stir both in theory and in practice. The concept of pursuing modernist design has influenced a number of designers and the design field has also expanded from the architectural field to other design fields and from Europe to the United States then elsewhere. It has also spawned many ideologically similar schools.Minimalism is to start from the idea of modernistic design and combine other relevant style at that time and come out. After 1950s, it also absorbs the principle of "postmodernism" in artistic creation and integrates the acceptable features.

Around 1945, the idea of minimalism appeared in the fields of clothing design, product design, and architectural design, graphic design, packaging design and so on, which was greatly promoted and spread. The term "Minimalism" made up of "minimal" and "ism" was established in 1960. Minimalism became popular in the world from the 1980s. It became a more influential design style which was widely used in the field of design.Although "Deconstruction" tried to negate this design style in the later 10 years, people hoped to find a calmness and convenience in daily life. Therefore, whether in internal meaning or external form, the simple style has adapted to the new aesthetic values germinated in such an environment. At the end of last century, minimalism touched on the field of graphic design and developed rapidly.

\section{Problems and Development Trend in Modern Packaging Design}

\subsection{Problems in Modern Packaging Design}

In China, the research on packaging design is very late, but the development of packaging design is very rapid.At present, the external form of packaging design has entered the multivariate era, and the countless strange packaging design has become a disaster.Some good works may leave a deep impression on consumers, but there are also some works with various problems.Among these problems, the most serious ones are usually in two forms: excessive packaging and excessive pursuit of form. This is also the most frequent problem in recent years.

Excessive packaging design, exquisite but not practical.It not only wastes ecological resources, but also harms consumers' trust, because the recycling rate of some packaging materials is not high, which cannot be treated and decomposed as garbage.For example, we often saw several small moon-cakes of low value are packaged in more than a dozen high-end gift boxes on the Internet or on television in recent reports on sky-high moon-cakes. Sometimes it even used expensive materials to make a box that has no real meaning except for its function of storage. In addition, some packaging design not only has excessive packaging, but also has too much emphasis on formalism, this is a sick packaging concept.Although thousands of designs are attractive to people's vision, these packaging designs will be eliminated sooner or later in today's increasingly people-oriented world.Our country is vigorously advocating the building of an environment-friendly and energysaving society. These packaging design problems have been paid more and more attention by people, and the government and the design community have also put these issues on the agenda.

\subsection{The Development Trend of Modern Packaging Design}

Facing so much packing problem, the government and the market as well as the design also put forward many ideas for this purpose, these concepts in the later development of packaging design will have the effect of some guidance, packaging design is slowly on the right track now, some of the right trend is gradually dominate the packaging design.For example:

Green design. Green packaging design refers to the packaging design that causes little or no damage and pollution to the earth environment, does no harm to human life, and can be recycled and can promote sustainable development.

Rational design. The successful packaging design is not to cover the gold plated silver, the higher the better, but to design with the value of the thing itself, the buyer's pursuit, the differences in purpose of use. What we need is the most refined form, the most ergonomic structure and the most suitable raw materials.

National design.Good design should have its own national traditional symbol. Excellent design should be integrated into the values of traditional culture, combined with current excellent design, 
and create its own characteristic brand, which is also the development advantage of our packaging design in China.

Humanized design. Excellent packaging should be based on ergonomics and center on product functions, stress on the design of human nature.It should meet the people-oriented requirements, including modeling, structure, color and other aspects.

\section{The Application of Minimalism in the Modern Packaging Design}

\subsection{Grasp Various Elements in Minimalism Packaging Design Accurately}

Integrating minimalism into packaging design, the most crucial part is to understand the products all-around.We should locate the product's consumer group according to the market research, then according to the characteristics of the product to design, including the elements such as structure, form and layout as well as the elements such as words, signs and images.

\subsubsection{The Embodiment of the Material Elements in the Minimalism}

In the minimalist style of packaging design, the use of elements must be combined with the user's requirements and the science and the technology. Generally, the common practice is about:(1)The effective use of natural elements. The natural straw packaging from Japan uses natural straw. It can not only reflect the beautiful natural style of product packaging but also reduce pollution and protect the environment.(2)The effective use of recycled materials.Recycled materials refer to materials that can be recycled or reused. For example, recycled paper, recycled plastic, recycled glass. The packaging materials used for some contemporary products are recycled paper. Recycled materials were being churned, recycled, and squeezed into a pulp model and then use it as a packaging. The principle of packaging design is that when certain resources or materials are not used, they will be recycled.

\subsubsection{The Expression of Characters and Symbols in the Minimalism}

In the minimalism, the meaning of words and symbols is to convey information. They make the information content accurate and striking in order to attract customers to buy impulsively.In packaging design, we should not only grasp the structure of the font, but also consider the layout of the text according to the various elements of the product, as well as the interaction between the font and the graphics.The form of font design is varied but it can not be complicated. The core idea of minimalism is to make the design concise and practical, so we must integrate the elements on the whole and rely on the characteristics of the product. We must use the most direct way to show the most powerful vision.In fact, some of the Chinese font designs on packaging have been well received by people especially the specialty products. The font design of this kind of products has been adopted by Chinese designers in traditional forms such as seal cutting, painting and calligraphy. It not only has authentic Chinese traditional forms, but also highlights the concept of minimalism.

\subsubsection{The embodiment of modeling and structural elements in minimalism}

Modeling and structure are two important links in packaging design and the structure directly affect the use of packaging.There are two main aspects of their performance:(1) Conciseness of modeling and structure.Minimalism is committed to the integration of simple and practical. It is most outstanding in the structure and other aspects. Simple structure is reasonable, simple shape is beautiful. This feature not only allows people to rediscover the design and aesthetic but also save resources effectively.(2) Reusable packaging.For example, after the use of iron tea box and mineral water bottle, its packaging can still be recycled. The iron tea box can be used as a money box and the mineral water bottle can be turned into a pot container by pruning. These designs are the examples of reusable. These not only reduce the waste of resources, but also have a unique style. 


\subsubsection{The representation of graphic and color elements in minimalism}

In minimalism, we need to consider whether there is a visual distinction between the primary and secondary. In addition, it can not abandon its essential attributes, that is, using the most concise design to attract consumers. This is the most difficult place for minimalism.In the packaging design, in addition to the use of very simplified fonts, products, characters and animal images, minimalism also needs to be fused and to show a great effect. The color is generally fused with graphics together.Color is also very important in minimalism. We should consider color collocation from two aspects: the first one is the product color, the second is the theme of the design.Research shows that using bright colors may has more sales than products using dark colors. Secondly, we need to know which colors are more pleasant. Finally, we should generalize colors to achieve a clear effect.

\subsection{The Rational Use of the Principle of Design in Minimalism}

\subsubsection{The Rational Use of the Functional Principles in the Minimalism}

The principle of functionalism is the primary principle of design. It can be said that design is to express "function" more effectively. On the contrary, the design will lose its essential meaning. The minimalist style of packaging design is pursuing the principle of "functional supremacy". It will put the idea of function in the first place no matter what kind of packaging, followed by other principles such as artistic principles. The functional principle of minimalism contains the idea of saving, which is the commonality of several styles of "functional supremacy". This idea requires minimalist designers to have a reasonable grasp of "degree". Of course, there are other more profound details about minimalism.

\subsubsection{The Rational Use of the Artistic Principle in the Minimalism}

The artistic principle includes the aspects of shape, color and so on. A package has the appropriate aesthetic characteristics and high artistic taste, which can give consumers the enjoyment of beauty. The principle of artistry can guide designers to handle the shapes of works skillfully. The art advocated by minimalism is not superficial, but an organic unity of the essential elements and forms of the product. Excellent art form can upgrade the product to a professional level and the connotation of the product will also be improved. Of course, art should also show the "degree" and the scientific including color, texture, modeling and so on. Therefore, a truly excellent product packaging must be a perfect combination of function and form.

\subsubsection{Rational Use of the Principle of Innovation in Minimalism}

Today's society is an innovative society, which is also strongly advocated by the state.Under such preconditions, more and more design schools and design fields have put design innovation on the agenda. First of all, it should be the innovation of the concept of design. We must break the past educational mode and traditional thinking. Secondly, we should innovate in practice. The design is facing the market and various kinds of science and technology emerge in endlessly. The design can not be limited to traditional materials, shapes, colors or technologies. Minimalism is a kind of innovation. Its ideas, materials and manufacturing processes all pursue the innovation. For example, to create a minimalist architecture and furniture, we are not just looking for white houses, white walls and simple furniture. It is more important to emphasize the overall structure and it uses the most advanced and convenient area division and furniture creativity as well as the humanized decoration which is more suitable for people's life, this is the kernel of the innovation in simplicity.

\subsubsection{Rational Use of the Economic Principle of Minimalism}

Nowadays, it will talk about economic principles when it comes to business. Of course, there is also a part of the view that some high quality design does not need to consider the economic factors with the improvement of people's quality of life, which is not correct. First, the packaging of minimalism inherited the characteristics of "cost saving" in modernist design. Again, minimalism has advanced ideas - frugality. Of course, many modern designers choose to serve the people. Excellent design is no longer a privilege enjoyed by aristocrats. Therefore, in modern packaging 
design, we must not neglect economic factors. Minimalism must take economic factors into account. Economic factors including the cost of production, the life of packaging materials and materials should be considered in modern excellent design. In the design process, the most practical and most valuable packaging design can be exchanged with low cost.

\subsubsection{Rational Use of the Informational Principle in Minimalism}

Because of the policy information, market information, technical information and other information have become important conditions for the success of the design, the information principle is becoming more and more important in the future design. Packaging designers should also pay attention to all kinds of information outside the product and use these information purposefully, then they also should consider the consumer's identity, status, character, culture and other information. Of course, its premise is to ensure the function of the product, otherwise it will go in the opposite direction. Minimalism must be responsive to the latest information of the age and integrate it effectively with other elements. At the same time, we should also master more advanced technology to promote the progress of materials, colors, processing and manufacturing in minimalism. For example, Chinese modern designers have designed a combination of Chinese and Western Font elements, which is benefited from the exchange of culture and the advent of the information age.

\section{Conclusion}

With the development of commodity economy, packaging design becomes more and more important in people's life and it also has many problems. The combination of minimalism and packaging design can effectively solve some problems and lead the trend of brief. The practical and concise concept advocated by minimalism has become the revolution of packaging design and it has created a new mode and trend. This consumption of packaging design is popular in both high consumption area and ordinary consumption area, which has been promoted widely.

\section{References}

[1] Yu Qiang, Introduction to Design [M], Chongqing: Chongqing University Press, 2006.8.1:45-46

[2] Ma Yongjian, 20 Lectures on Modern Art [M], Shanghai: Shanghai Academy of Social Sciences Press, 2005.10:4-7

[3] Zhang Daoyi, Designed for Mou[M],Chongqing: Chongqing University press,2007.5.30:21

[4] Li Yanzu, Product Design Art [M], Beijing: Renmin University of China press, 2005.1:40-41

[5] Tang Hua, Visual Arts Theory [M], Beijing: Capital Normal University press, 2008.10.1:29-30 